\title{
Recall costs balanced against spoilage control in Dutch custard
}

\author{
A. G. J. Velthuis, ${ }^{* 1}$ M. W. Reij, $†$ K. Baritakis, ${ }^{\star} \dagger$ M. Dang, ${ }^{*}$ and C. P. A. van Wagenberg $\ddagger$ \\ *Business Economics, Wageningen University, Hollandseweg 1, 6706 KN, Wageningen, the Netherlands \\ †Food Microbiology, Wageningen University, Bomenweg 2, 6703 HD, Wageningen, the Netherlands \\ fLEI Wageningen UR, Wageningen University and Research Centre, Hollandseweg 1, 6706 KN, Wageningen, the Netherlands
}

\section{ABSTRACT}

The relation between the moment at which a recall of Dutch custard is initiated and the direct costs of this recall was investigated. A simulation model of the custard supply chain was developed to compare scenarios with and without a quarantine of $48 \mathrm{~h}$ at the storage of the production plant. The model consists of 3 parts: 1) the distribution of a 24,000-L batch of custard over the supply chain over time is simulated; 2) the time to detect spoilage bacteria with a recontamination test procedure is simulated; and 3) the direct recall costs of custard over the different parts of the supply chain are calculated. Direct recall costs increase from about $€ 25,000 /$ batch to $€ 36,171 /$ batch from 57 to $135 \mathrm{~h}$ in the situation without quarantine and from $€ 25,000 /$ batch to $€ 36,648 /$ batch from $123 \mathrm{~h}$ to $163 \mathrm{~h}$ for the situation with quarantine. Then costs decrease because more and more custard is at the consumer level and only $0.13 \%$ of the consumers will ask for a refund. With low true contamination probabilities quarantine is not profitable, but at later detection moments with high probabilities it is. We conclude that a simulation model is a helpful tool to evaluate the efficiency of risk management strategies like end product testing and a quarantine situation.

Key words: quality control, custard, failure cost, recall cost

\section{INTRODUCTION}

If control during food processing fails and unsafe food products have reached the market, these products must be withdrawn from downstream businesses and must be recalled from the consumer (Article 19; EC, 2002). Food processors in the European Union are responsible for recalls of unsafe food products and bear the financial consequences of the recalls, which can be quite significant (Skees, 2001).

\footnotetext{
Received August 18, 2009.

Accepted March 1, 2010.

${ }^{1}$ Corresponding author: annet.velthuis@wur.nl
}

Next to legally required recalls for unsafe food, companies can decide to recall their products if a quality deficiency is detected that will not pose any health risk to the consumer but may give rise to consumer complaints. We will call this defective food in this paper. The decision of a food processor to initiate a recall of defective food products depends on the costs of the recall, on the financial consequences of direct market problems that defective products create, and on the negative long-term effects on profitability of the product and the related brands. Processors will not execute a recall if the costs outweigh the benefits.

Direct costs of a recall include costs for media announcements, transportation, warehousing costs, extra labor, and destruction (Meuwissen, 2009; Velthuis et al., 2009). Indirect recall costs include reduced sales and revenues, a lower stock price in the capital market, costs incurred for brand rehabilitation, and crisis response expenses (Salin et al., 2005). Brand rehabilitation might be necessary to reestablish the reputation and market share of the affected product by investing in advertising campaigns, special promotions, and consumer education. Crisis response expenses include fees and expenses of outside consultants retained exclusively for the function of responding to the product contamination and recall. The indirect costs of a recall are difficult to quantify (Kramer et al., 2005).

Early initiation of a recall minimizes indirect costs and helps to maintain consumer confidence (Meuwissen et al., 2003). An efficient monitoring system can shorten the period between the detection of the problem and the execution of a recall (Teratanavat and Hooker, 2004). In addition, an effective traceability system, which provides data about the (previous) locations of food and food ingredients of each batch along the production chain, can also shorten this period (Meuwissen et al., 2003; Dorp, 2004). Finally, a smaller batch size, which is the quantity of products produced under uniform conditions, results in lower recall costs (Dupuy et al., 2005), although, because of economies of scale, the effect might be small.

The moment of detection of a problem is an important factor for the costs of a recall. However, the quan- 
titative relation between this moment and recall costs has only recently received some attention and is still insufficiently understood. This relationship has been quantified only for spoilage of consumption milk, which is a simple product in terms of having 1 ingredient and only a few steps in the production process (Velthuis et al., 2009). Velthuis et al. (2009) did not focus on one food safety or food quality problem, and nor did they consider the detection speed of the monitoring program. In the current study we extend the existing knowledge by applying the simulation approach to spoilage of a more complex product (i.e., custard) and by including the detection speed of the monitoring program.

Dutch custard (or "vla" in Dutch) was chosen because it is a homogeneous product with a limited number of ingredients and straightforward processing. Dutch custard is a sweet dairy dessert that can be poured from a pack with a viscosity similar to yogurt. It is produced by mixing milk, sugar, flavors, colorants, and thickeners to form a liquid pudding. After mixing, the custard is pasteurized at a temperature of around $100^{\circ} \mathrm{C}$ to inactivate all bacteria except for some thermoduric organisms and spores. The pasteurized product is stored for a limited period in silos and finally is packed under aseptic conditions.

Microbial spoilage in sweet, nonacidified dairy products such as custard results either from gram-positive bacteria, which can survive pasteurization, or from any psychrophilic, "cold-loving" gram-negative bacteria that contaminate the product after the heat treatment. Most prevalent gram-positive bacteria in dairy products are Bacillus cereus (Eneroth et al., 2000; Huck et al., 2008), and most prevalent gram-negative bacteria are Pseudomonas species.

Spoilage caused by Pseudomonas spp. bacteria leads to the development of off taste and off odors. Under refrigerated and frozen conditions the growth rate of Pseudomonas spp. is much higher than the growth rate of gram-positive bacteria. Consequently, the onset of spoilage is much earlier in packs contaminated with Pseudomonas spp. than in packs that contain grampositive bacteria only. Pseudomonas spp. can be regarded as an indicator for any pathogenic or nonpathogenic heat-sensitive microorganisms that have gained access to the product after the pasteurization step. For a dairy processor the main risk of Pseudomonas spp. contaminations is not food safety but that the retailers and consumers stop buying their custard. This study focuses on postprocessing contamination with Pseudomonas spp., which originates from unclean lines or tanks downstream of the pasteurizer, the environment during filling, or defective packages.

To avoid the sale of products that are spoiled before the use-by date (which is $25 \mathrm{~d}$ or $600 \mathrm{~h}$ after pasteuriza- tion) because of the presence of Pseudomonas spp., dairy companies implement rigid maintenance, cleaning, and sanitation programs and monitor the microbiological quality of the final product, the so-called end product testing. A further strategy is to implement a quarantine situation. In a quarantine situation, a batch of custard leaves the plant after the microbiological test results of that specific batch have shown no contamination. Eneroth et al. (1998) found that a quarantine period of $48 \mathrm{~h}$ is sufficient to identify a postpasteurization contamination by Pseudomonas spp.

This paper aims to investigate the relation between the time at which the results of end product testing become available and a recall may have to be initiated, and the direct costs of such a recall. We focus on the direct recall costs because the mechanisms that determine the indirect recall costs are unknown and empirical information about these costs is lacking. We compare 2 scenarios: with and without a quarantine situation of $48 \mathrm{~h}$.

\section{MODEL DESCRIPTION}

The model consists of 3 parts. The first part simulates the distribution of a batch of custard over the supply chain over time (custard supply chain model), the second part simulates the time it takes to fulfill the recontamination test procedure to detect spoilage bacteria (end product testing model), and the third part calculates the direct recall costs of a batch of custard in each part of the supply chain (recall cost model).

\section{Custard Supply Chain Model}

The custard supply chain model simulates the amount of custard in each step in the supply chain at a certain moment in time. This model is a Monte Carlo simulation. It considers the flow of an average batch of Dutch custard in the supply chain from the production plant up to and including consumers (Figure 1). A batch is defined as all the packages produced and packed under similar conditions containing the same unique identification code, which includes production line number and use-by date.

The stochastic variables of the model are the length of stay of each subbatch of custard in each step in the chain (Table 1). The length of stay is modeled with a triangular distribution described by a minimum value, a most likely value or mode, and a maximum value (Vose, 2000). The triangular distribution is typically used as a description of an event for which there is limited data. A triangular distribution with its input based on expert opinion is a reasonable approximation of the actual value. The Monte Carlo simulation model 


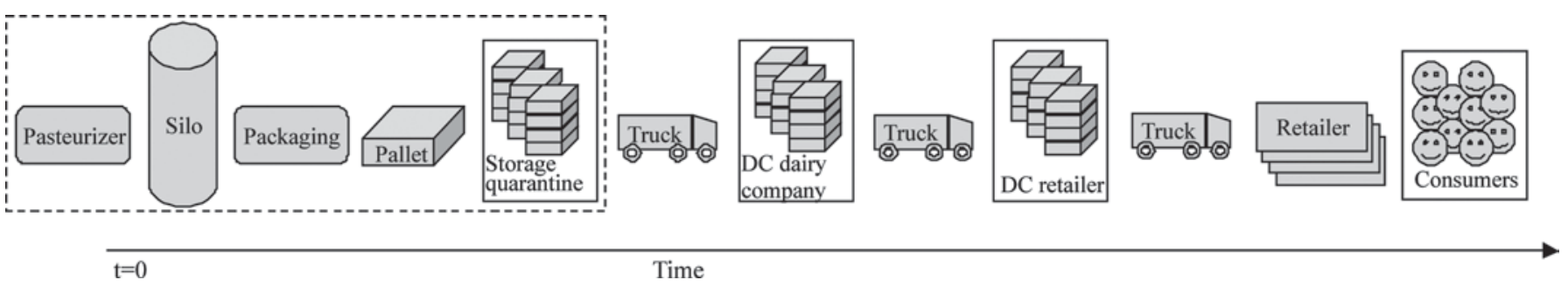

Figure 1. The Dutch custard production chain. DC = distribution center. Color version available in online PDF.

was built in Excel 2003 (Microsoft Corp., Redmond, WA) with the add-in program @Risk 4.5 (Palisade Corporation, 2004).

The input values in Table 1 describe the distributions of each step in the supply chain. The values used in the calculations were determined with experts from a Dutch dairy cooperation. Monte Carlo simulation allows drawing for each subbatch a time value from the corresponding triangular distribution (Vose, 2000). For example, if the first number drawn from the triangular distribution that describes filling the silo is 2.52 , this means that the first silo with a content of $17,000 \mathrm{~kg}$ is filled in $2.52 \mathrm{~h}$ after the custard has passed the high pasteurization step.

The supply chain in our model starts after the pasteurization in the production plant at time $(\boldsymbol{t})=0$ and ends at the moment the custard is consumed at the consumer's home. The processing line before pas- teurization is not considered because Pseudomonas spp. bacteria are effectively killed by pasteurization and the initial number of colony forming units at $t=0$ can therefore be assumed to be negligible.

The average batch size is $24,000 \mathrm{~L}$. After pasteurization the custard is simultaneously pumped into 2 silos, each with a capacity of $17,000 \mathrm{~L}$. Therefore, to produce 1 batch of custard, the contents of 1.4 silos are needed. The custard stays in the silos for some hours depending on the planning of the filling machines. Then the custard is pumped into filling machines in simulated subbatches of $50 \mathrm{~L}$, where packages $(1 \mathrm{~L})$ are filled. During the filling step, 4 packages are filled simultaneously. It takes around $3 \mathrm{~min}$ to pump $50 \mathrm{~L}$ into the filling machine and fill the packages.

Once filled, the packages are moved to and put on a pallet. This procedure takes a few minutes. The moment the last package (i.e., package number 720) is put

Table 1. Input of the simulation model: distribution characteristics of the length of stay, the number of liters per draw, and number of draws (based on a 24,000-L batch of custard) of each separate step in the custard chain in the model

\begin{tabular}{|c|c|c|c|c|c|c|}
\hline \multirow[b]{2}{*}{ Step in the Dutch custard chain } & \multirow[b]{2}{*}{ Distribution } & \multicolumn{3}{|c|}{ Length of stay (h) } & \multirow[b]{2}{*}{$\mathrm{L} /$ draw $(\mathrm{n})$} & \multirow[b]{2}{*}{ Draws $(n)$} \\
\hline & & Minimum & Mode & Maximum & & \\
\hline Filling the silo & Triangular & 2.00 & 2.50 & 3.00 & $17,000.00$ & 2 \\
\hline Storage in the silo & Triangular & 5.00 & 6.00 & 7.00 & 50.00 & 480 \\
\hline Flowing from silo to filling machine & Triangular & 0.01 & 0.05 & 0.08 & 50.00 & 480 \\
\hline Flowing from filling machine to pallet ${ }^{1}$ & Triangular & 0.05 & 0.06 & 0.22 & 4.00 & 6,000 \\
\hline Time until full pallet $\left(720\right.$ packages) ${ }^{2}$ & - & - & - & - & - & - \\
\hline Moving full pallet to cold storage & Triangular & 0.08 & 0.13 & 0.25 & 720.00 & 34 \\
\hline Storage in cold storage (without quarantine) & Triangular & 0.50 & 48.00 & 96.00 & $7,200.00$ & 4 \\
\hline Storage in cold storage (with quarantine) & Triangular & 48.50 & 96.00 & 144.00 & $7,200.00$ & 4 \\
\hline Time until a full truck (11 pallets) ${ }^{3}$ & - & - & - & - & - & _ \\
\hline Transport from storage to dairy company $\mathrm{DC}^{4}$ & Triangular & 1.00 & 1.50 & 2.00 & $7,200.00$ & 4 \\
\hline Storage in dairy company DC & Triangular & 3.00 & 36.00 & 72.00 & $10,800.00$ & 3 \\
\hline Transport from dairy company DC to retailer DC & Triangular & 1.00 & 3.00 & 5.00 & $10,800.00$ & 3 \\
\hline Storage in retailer DC & Triangular & 1.00 & 8.00 & 12.00 & $8,000.00$ & 3 \\
\hline Transport from retailer DC to retailer stores & Triangular & 1.00 & 4.00 & 12.00 & 480.00 & 50 \\
\hline Storage in retailer store & Triangular & 2.00 & 12.00 & 72.00 & 480.00 & 50 \\
\hline Storage at consumer until consumption ${ }^{1}$ & Triangular & 1.00 & 36.00 & 168.00 & 4.00 & 6,000 \\
\hline
\end{tabular}


on a pallet, is used in the model as the starting time for moving this pallet to the cold storage. From this moment a new empty pallet is filled. The starting time for this second pallet to be moved to the cold storage is the moment package 1,440 is put on the pallet. This is repeated until all 6,000 packages are put on pallets. In total, 33 and one-third pallets are filled.

In the model we distinguish between 2 options for the dairy company. First, in the situation without quarantine, the dairy company moves the products further in the chain even if test results are not available. Second, in a situation with quarantine, most batches of products leave the plant after the test results of that specific batch have shown no contamination. We assume a quarantine period of $48 \mathrm{~h}$ because this should be sufficient to identify a possible postpasteurization contamination by Pseudomonas spp. (Eneroth et al., 1998).

After the cold storage, the batch, together with batches of other products, is transported with 4 trucks to the distribution center (DC) of the dairy company. We assume that the first 3 trucks each carry 11 pallets of this batch and the fourth truck carries one-third of a pallet of this batch.

The pallets stay in the DC of the dairy company before being transported to the DC of the retailers' organization. Until the moment that a truck with the custard leaves the DC of the dairy company, the dairy company is responsible for the custard. If this moment exceeds $8 \mathrm{~d}$ (or $192 \mathrm{~h}$ ) the retailer will reject the (sub) batch and the custard will be destroyed.

The batch of custard is transported with 3 trucks, of which the first 2 trucks each carry 15 pallets and the last truck carries 3 and one-third pallets. Each drive takes between 1 and $5 \mathrm{~h}$, depending on the location of the DC of the retailer's organization and the traffic. After arriving at the DC of the retailer's organization the batch is divided over 50 retailer trucks, each carrying $480 \mathrm{~L}$ of custard of the batch (next to other items) to a specific supermarket. Once the custard has arrived in a supermarket it is sold. Each consumer buys 4 packages.

The custard supply chain model predicts the amount of custard in each step in the supply chain at the moment a recall is initiated. This moment is defined as the recall moment and is presented with $t$, the number of hours after the moment at which the first custard leaves high pasteurization $(t=0)$. The distribution of the amount of custard in each step in the supply chain is simulated with 1,000 recalls of custard batches. This distribution is used to calculate the direct recall costs per step and of the whole supply chain.

\section{End Product Testing Model}

In the end product testing model the time to detect a contamination with Pseudomonas spp. in a batch of custard is simulated and the probability of detection given a true probability of contamination is calculated.

The assumed test protocol is as follows. Of the final product, 16 packs are taken randomly from the production line while they are put on a pallet to be tested for the presence of Pseudomonas spp. Note that this moment is simulated and depends on the length of stay of the product in the steps before this point. The 16 packages are stored in a refrigerator at $<7^{\circ} \mathrm{C}$ at the production site until $0900 \mathrm{~h}$ the next day, when they are transported to an external laboratory. When production is on Saturday the packages remain in the refrigerator an additional $24 \mathrm{~h}$ until the packages are transported Monday morning. The storage time in the refrigerator has a uniform distribution over 1 to $24 \mathrm{~h}$, supplemented with a binomial distribution with a probability of 1 in 7 for an additional $24 \mathrm{~h}$ for products produced on Saturday.

In the laboratory the packages are stored in a refrigerator at $<7^{\circ} \mathrm{C}$ for $8 \mathrm{~h}$ until the end of the working day at $1800 \mathrm{~h}$, when they are placed into a $30^{\circ} \mathrm{C}$ incubator for a fixed preincubation period of $14 \mathrm{~h}$. Preincubation allows any microorganism that may have contaminated the package to multiply to numbers that can be detected in the next step. The packages are left in the incubator for $1 \mathrm{~h}$ until the laboratory staff starts working at $900 \mathrm{~h}$. After opening the preincubated packages, $50 \mu \mathrm{L}$ of each package is inoculated onto agar plates with a medium that is selective for gram-negative bacteria, followed by incubation at $30^{\circ} \mathrm{C}$ and reporting of the results to the factory. Inoculation, incubation, and reporting require 1,24 , and $1 \mathrm{~h}$, respectively and are assumed to be fixed. The time between reporting to the quality assurance manager and taking action in case of a recall has a triangular distribution and is assumed to be between 0 and $3 \mathrm{~h}$, with a most likely value of $0.5 \mathrm{~h}$.

\section{Probability to Detect Contamination}

The probability to detect at least 1 contaminated package of custard $[\operatorname{Pr}(\boldsymbol{P S} \geq \mathbf{1})]$, given a certain fraction of products in a batch that are truly contaminated, the so-called true contamination probability $[\operatorname{Pr}($ cont $)]$, and several samples taken ( $n$; i.e., 16 packages/batch), is calculated as

$$
\operatorname{Pr}(P S \geq 1)=1-[1-\operatorname{Pr}(\text { cont })] .
$$


We assume that a recall will be performed if 2 or more samples are tested positive in the end product test $[\operatorname{Pr}(\boldsymbol{P S} \geq \mathbf{2})]$. It is calculated as

$$
\operatorname{Pr}(P S \geq 2)=\operatorname{Pr}(P S \geq 1)-\operatorname{Pr}(P S=1),
$$

where

$$
\begin{gathered}
\operatorname{Pr}(P S=1)=n \times \operatorname{Pr}(\text { cont }) \\
\times\left\{1-[1-\operatorname{Pr}(\text { cont })]^{n-1}\right\},
\end{gathered}
$$

in which $\operatorname{Pr}(P S=1)$ is the probability of detecting exactly 1 positive sample. For the analysis, $\operatorname{Pr}($ cont $)$ is varied over the range of 0.005 to 0.100 to reflect both the normal situation where incidental postprocessing recontamination occurs and the rare situations where biofilms cause contamination of a large percentage of the packages.

\section{Calculation of the Recall Costs}

The direct recall costs are calculated following the partial budgeting approach. This approach is based on the principle that a small change in the supply chain can eliminate or reduce some costs, eliminate or reduce some returns, cause additional costs to be incurred, and cause additional returns to be received. The net effect of the change, in our case the recall, is the sum of the positive financial impact minus the sum of the negative financial impact (Dijkhuizen and Morris, 1997):

$$
N C_{r}=A C_{r}+R R_{r}+R C_{r}+A R_{r},
$$

where $N C_{r}$ is the net costs of the recall, $A C_{r}$ is the additional costs, $R R_{r}$ is the reduced returns, $R C_{r}$ is the reduced costs, and $A R_{r}$ is the additional returns in the whole supply chain.

The $A C_{r}$ include various activities and is given by

$$
\left\{\begin{array}{ll}
A C_{r}=\sum_{i}\left(T_{i}+D_{i}+R_{i}+C_{i}\right) & \\
\times B S_{i}+\sum_{j} I_{j}+\sum_{k} L_{k} & \text { if } B S_{t r 2}<0 \text { and } t \leq 600 \\
A C_{r}=\sum_{i}\left(T_{i}+D_{i}+R_{i}+C_{i}\right) & \\
\times B S_{i}+\sum_{j} I_{j}+\sum_{k} L_{k}+M A+R F & \text { if } B S_{t r 2} \geq 0 \text { and } t \leq 600 \\
A C_{r}=\sum_{j} I_{j} & \text { if } t>600
\end{array},\right.
$$

where $T$ is the transportation costs including the warehousing costs, $D$ is the destruction costs of the packed custard, $R$ is the fee to pay a feed company for reusing the unpacked custard, $C$ the cleaning costs of the production lines including labor, and $B S$ is the amount of the recalled batch that is located in the $i$ th step in the chain (Table 1). The additional inspection costs $(I)$ differ among internal, third party, and governmental inspections, indicated by $j$. The extra labor $(L)$ within the dairy company depends on task $k$ : handling the recalled products, crisis management, and labor for the consumer care line. We assume that the moment the first truck leaves the DC of the dairy company to the $\mathrm{DC}$ of the retailer's organization, indicated by $B S_{t r 2}$, the recall becomes public and additional costs occur. The additional costs include the costs of a media announcement $(M A)$ and the costs of refunds the consumers who bought a package of the recalled batch $(R F)$. If $t$ exceeds the expiration date (which is $25 \mathrm{~d}$ or 600 $\mathrm{h}$ after pasteurization), only the costs of additional inspections remain.

The costs $M A$ are based on criteria of the Dutch Food and Consumer Product Safety Authority of advertisements in at least 2 national newspapers, an official press release, and an announcement on the Web site of the producer, and are calculated as

$$
M A=P R+\left(p r_{a d} \times a d s\right)+\text { others },
$$

where $P R$ is the costs for the dairy company to hire an external public relation specialist who makes the advertisements and a press release for €300, $p r_{a d}$ is the costs of placing an advertisement in a national paper, ads is the number of advertisements in national newspapers, and others is the costs of sending the press release to customers and governmental organizations and for the announcement on the producer's Web site.

The costs $R F$ include the price of a postage stamp $\left(p r_{\text {stamp }}\right)$ and the consumer price of a package of custard $\left(p r_{\text {cons }}\right)$, multiplied by the percentage of consumers that returned barcodes of packages from the recalled batch (cons) and the amount of custard that is sold to consumers and not consumed $\left(B S_{\text {cons }}\right)$ :

$$
F=\left(p r_{\text {stamp }}+p r_{\text {cons }}\right) \times \text { cons } \times B S_{\text {cons }} .
$$

Few packages are usually sent back in the case of a public recall. Based on a former recall, where 20 barcodes out of 15,000 were sent back, we assumed that $0.13 \%$ of packages bought are returned for a refund (cons).

The reduced returns $\left(R R_{r}\right)$ of the dairy company include the forgone returns of the custard that replaces 
Table 2. Description and values of the variables in the net costs calculations of the recall costs of 1 batch of custard

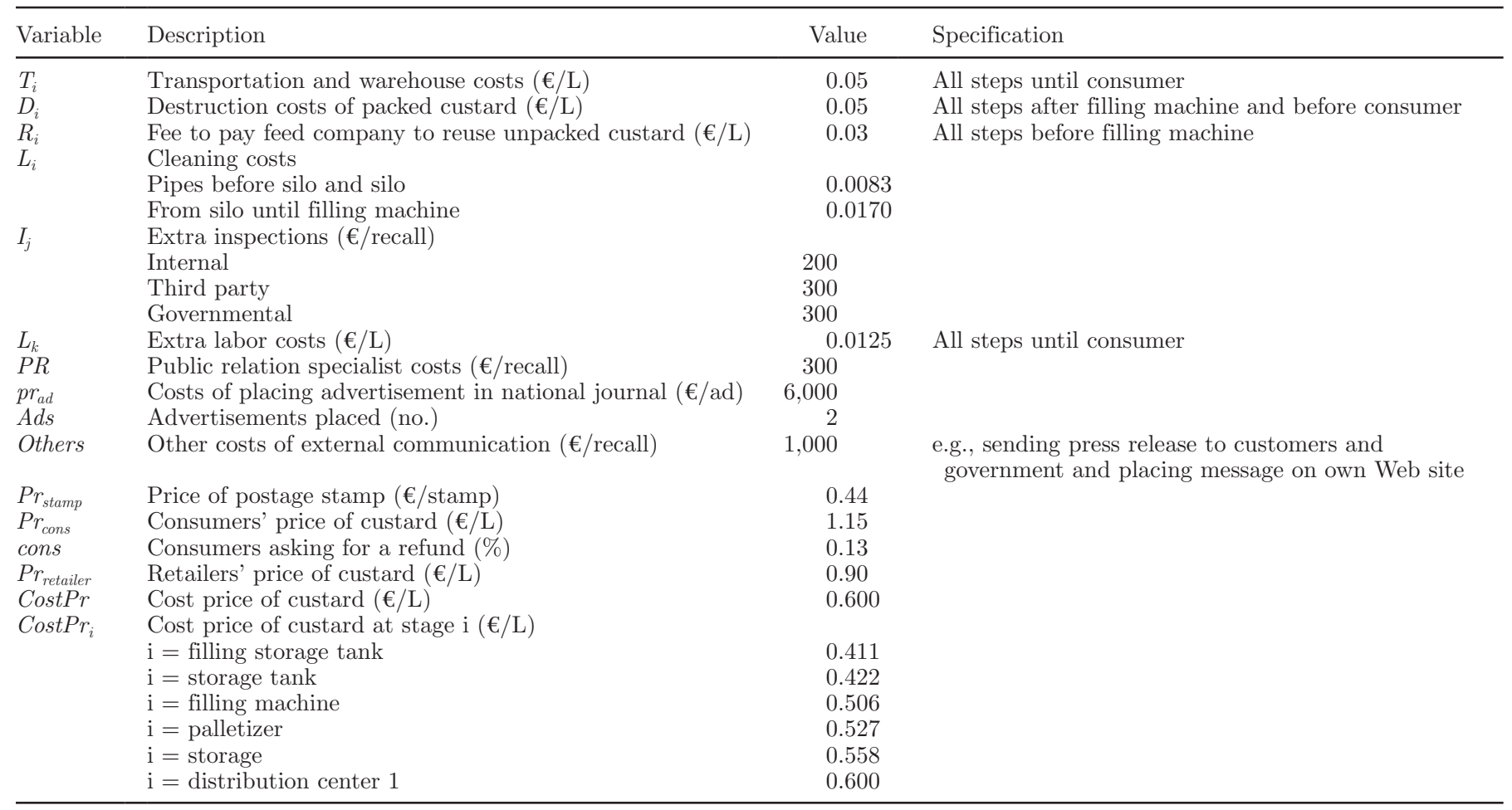

the recalled batch that was recalled at the retailers. The reduced returns equal the price $\left(p r_{\text {retailer }}\right)$ the dairy company receives from the retailer:

$$
R R_{r}=p r_{\text {retailer }} \times B S_{\text {retailer }}
$$

The reduced costs $\left(R C_{r}\right)$ of the dairy company are the costs that do not have to be made in the rest of the chain for the part of a recalled batch that is still located somewhere in the supply chain. For example, custard of a recalled batch that is still located in the storage of the production plant at the initial moment of a recall does not have to be shipped to and stored in the DC of the dairy company and retailer. These costs are the difference between the cost price of the product (CostPr) of the end product in the supermarket and the costs $\left(C_{\text {ost } P r_{i}}\right)$ made until step $i$ :

$$
R C_{r}=\sum_{i}\left(\operatorname{CostPr}-\operatorname{CostPr}_{i}\right) \times B S_{i}
$$

We assumed that there are no additional returns $\left(A R_{r}\right)$ when recalling a batch of custard, although the recalled custard can be used for the production of animal feed. Depending on the market of feed materials, the dairy company may receive additional returns for the batch from the feed producer. In this study we assumed that the dairy company has to pay the feed producer to reuse the spoiled batch. Furthermore, we assumed that the dairy company has no recall insurance and receives no insurance payments.

Table 2 gives the values of the different input variables for the economic model. The data have been assessed in cooperation with a Dutch dairy cooperation.

The recall cost per average batch of custard produced $(\boldsymbol{A R C})$ shows how much failure costs because of spoilage should be taken into account when producing custard. The $A R C$ is calculated by multiplying the total recall costs $(N C)$ of a recalled batch by the probability that a recall will be performed [i.e., if 2 or more samples are tested positive in the end product test; $\operatorname{Pr}(P S \geq$ $2)]$ :

$$
A R C=N C \times \operatorname{Pr}(P S \geq 2) .
$$

\section{Calculation of Costs for Applying a Quarantine Situation}

The net costs of applying a quarantine situation are calculated following the partial budgeting approach:

$$
N C_{q}=A C_{q}+R R_{q}+R C_{q}+A R_{q},
$$

where $N C_{q}$ is the net costs, $A C_{q}$ is the additional costs, $R R_{q}$ is the reduced returns, $R C_{q}$ is the reduced costs, 
Table 3. Input values for the calculation of the additional costs of a quarantine situation

\begin{tabular}{llrc}
\hline Variable & Description & $€ /$ batch & $€ / \mathrm{L}$ \\
\hline$B C$ & Cost of extra storage space & 6.67 & 0.001 \\
& Maintenance of extra storage & 0.33 & 0.001 \\
$C C$ & Cooling to $4^{\circ} \mathrm{C}$ & 2.00 & 0.001 \\
$L_{q}$ & Labor & 150.00 & 0.001 \\
$\Delta p r_{\text {retailer }}$ & Reduction in retailer's price & 240.00 & 0.01 \\
\hline
\end{tabular}

and $A R_{q}$ is the additional returns if applying a quarantine situation compared with a situation without quarantine.

The $A C_{q}$ of a quarantine situation include the costs of building or renting additional storage space and maintenance of this space $(B C)$, cooling of the additional storage space to $4^{\circ} \mathrm{C}(C C)$, and additional labor costs $\left(L_{q}\right)$ :

$$
A C_{q}=B C+C C+L_{q} .
$$

The $R R_{q}$ of a quarantine situation is equal the lower retailer's price because of a reduced shelf life of $2 \mathrm{~d}$ $\left(\Delta p r_{\text {retailer }}\right)$ :

$$
R R_{q}=\Delta p r_{\text {retailer }} .
$$

There are $R C_{q}$ for applying a quarantine situation, whereas the $A R_{q}$ equal the reduction of the $A R C$ because of the quarantine situation $\left(A R C_{\text {quarantine }}\right)$ as compared with the nonquarantine situation $\left(A R C_{\text {no_quarantine }}\right)$ :

$$
A R_{q}=A R C_{\text {no_quarantine }}-A R C_{\text {quarantine }} .
$$

Table 3 provides the data for calculation of the net costs of applying a quarantine situation compared with a nonquarantine situation.

\section{RESULTS}

\section{Custard Supply Chain Model}

Figure 2 shows the distribution of 1 batch of custard over the supply chain in a situation without quarantine. Results are presented at the level of the owners being the dairy company, retailer, and consumers. Until 71 $\mathrm{h}$ after pasteurization the whole batch was still at the dairy company (i.e., not a single liter of custard left the $\mathrm{DC}$ of the dairy company before $71 \mathrm{~h}$ in all 1,000 simulated batches). If the recall moment was before $71 \mathrm{~h}$, it thus concerns an internal recall. One or more packages of a batch were at the retailer level between 71 and $275 \mathrm{~h}$ after pasteurization (3.0-11.5 d). The first package was bought by a consumer at $90 \mathrm{~h}$ and the last package at $431 \mathrm{~h}(3.8-18.0 \mathrm{~d})$ after pasteurization. The first custard was consumed at $97 \mathrm{~h}(4.0 \mathrm{~d})$ and the last custard at $432 \mathrm{~h}(18.0 \mathrm{~d})$ after pasteurization.

In a situation with quarantine the flow of custard through the chain is delayed at the production plant of the chain (Figure 3). The whole batch was at the dairy company level until $115 \mathrm{~h}$ after pasteurization (4.8 d). With quarantine, the packages of custard remained shorter at the retailer level compared with the nonquarantine situation [between 115 and $274 \mathrm{~h}$ (4.8-11.4 d)] and also remained shorter at the consumer level [between 134 and $433 \mathrm{~h}(5.6-18.0 \mathrm{~d})]$. The first package of custard was consumed at $138 \mathrm{~h}$ after pasteurization (41 $\mathrm{h}$ later than in the nonquarantine situation), whereas the last custard was consumed at $433 \mathrm{~h}$ after pasteurization $(18.0 \mathrm{~d})$. This is before the end of shelf life of $600 \mathrm{~h}$ after pasteurization.

\section{End Product Testing Model}

The minimum time to detect a contamination based on end product testing varies between 50.1 and $98.8 \mathrm{~h}$, with a mean value of $65.9 \mathrm{~h}$ (5th and 95th percentiles were 52.3 and $90.0 \mathrm{~h}$, respectively). The differences are attributable to the fact that samples collected on Saturday are sent to the laboratory on Monday and to the time the packages are stored in the refrigerator.

\section{Probability of a Recall}

The $\operatorname{Pr}(P S \geq 2)$ increases with the $\operatorname{Pr}($ cont) (Figure 4). If the true level of contamination is low (e.g., 0.005; $120 / 24,000$ packages contaminated), the probability to detect 2 or more positives and consequently to conduct a recall is low (0.003). This probability is 36 times higher (0.189) if the true level of contamination is 10fold higher $(0.050 ; 1,200 / 24,000$ packages $)$ and is 161 times higher (0.485) if the true level of contamination is 20 -fold higher $(0.200 ; 4,800 / 24,000$ packages $)$.

\section{Net Recall Costs}

The net recall costs of a contaminated batch of custard $\left(\boldsymbol{N} \boldsymbol{C}_{r}\right)$ depend on the recall moment as illustrated in Figure 5 for a situation without quarantine and in Figure 6 for a situation with quarantine. Net recall costs are around $€ 21,000$ if the recall moment is within $9 \mathrm{~h}$ after pasteurization. If the recall moment is 9 or more hours after pasteurization, the net recall costs are higher because from $9 \mathrm{~h}$ onwards some of the custard has been packaged and cleaning of the packaging line requires a lot of labor. However, because contamination is detected by the end product test and test results are with $90 \%$ certainty available from 52.3 to $90.0 \mathrm{~h}$ after 

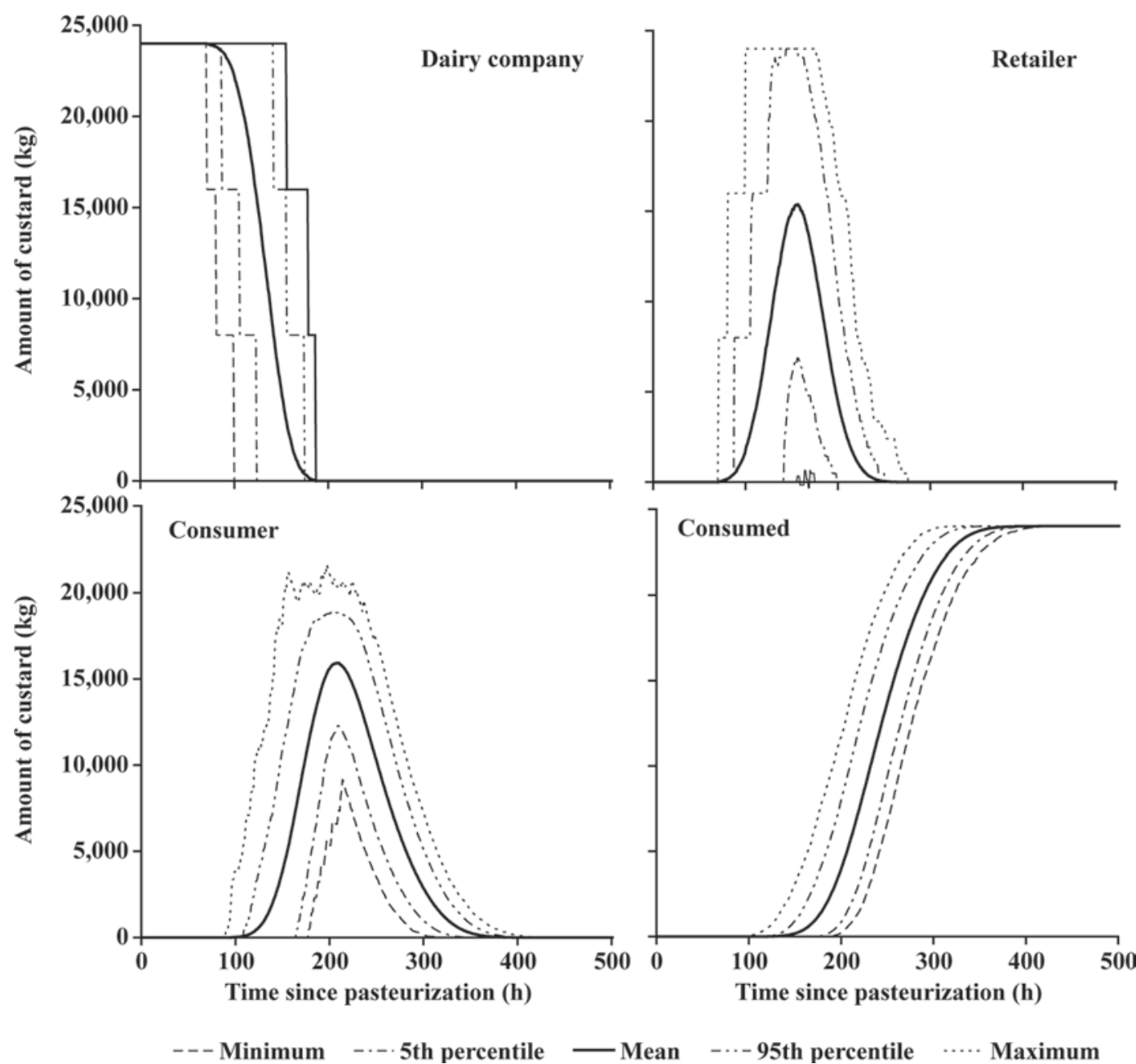

Figure 2. Location of the amount of custard of 1 batch $(24,000 \mathrm{~L})$ over the supply chain in a situation without quarantine. Top left panel: dairy company stage, including the production site, transport, and distribution center of the dairy company; top right panel: retailer stage, including the distribution center of the retailer, transport, and stores; bottom left panel: the consumer stage; bottom right panel: the consumed amount of custard.

pasteurization, the recall moment can be only in the interval as indicated with the gray areas in Figures 6 and 7.

The $N C_{r}$ values sharply increase around 70 and 110 $\mathrm{h}$ for the situation without and with quarantine, respectively. The increase in $N C_{r}$ is caused mainly by the costs of a media announcement related to a public recall $(€ 14,500)$. For the quarantine situation this means that the moment a recall is initiated by the end product test precedes the further increase of the mean total recall costs. This moment precedes even the further increase in the worst case scenario (maximum) if products move very fast through the chain. The $N C_{r}$ reach a maximum of $€ 36,171$ and $€ 36,648$ at 139 and $165 \mathrm{~h}$ for the nonquarantine and quarantine situation, respectively. After this moment, $N C_{r}$ decreases because more and more custard is at the consumer level and consumed.
This reduces $N C_{r}$ because only $0.13 \%$ of the consumers that detect spoilage will ask for a refund. Only the percentage sent in for refund induces direct costs to the company. However, additional company costs can occur if consumers that have detected spoilage stop buying their custard. In addition, consuming spoiled custard might lead to consumer illness with associated societal costs. In situations both without and with quarantine, the total recall costs from around $280 \mathrm{~h}$ onwards consist almost only of media announcement costs.

\section{Recall Costs per Average Batch of Custard}

The $A R C$ depends on the $\operatorname{Pr}($ cont $)$ because the $\operatorname{Pr}(P S \geq 2)$ depends on $\operatorname{Pr}($ cont) (Table 4). Also, ARC depends on the recall moment. Recall moments of 66 and 90 were chosen because they reflect the mean and 

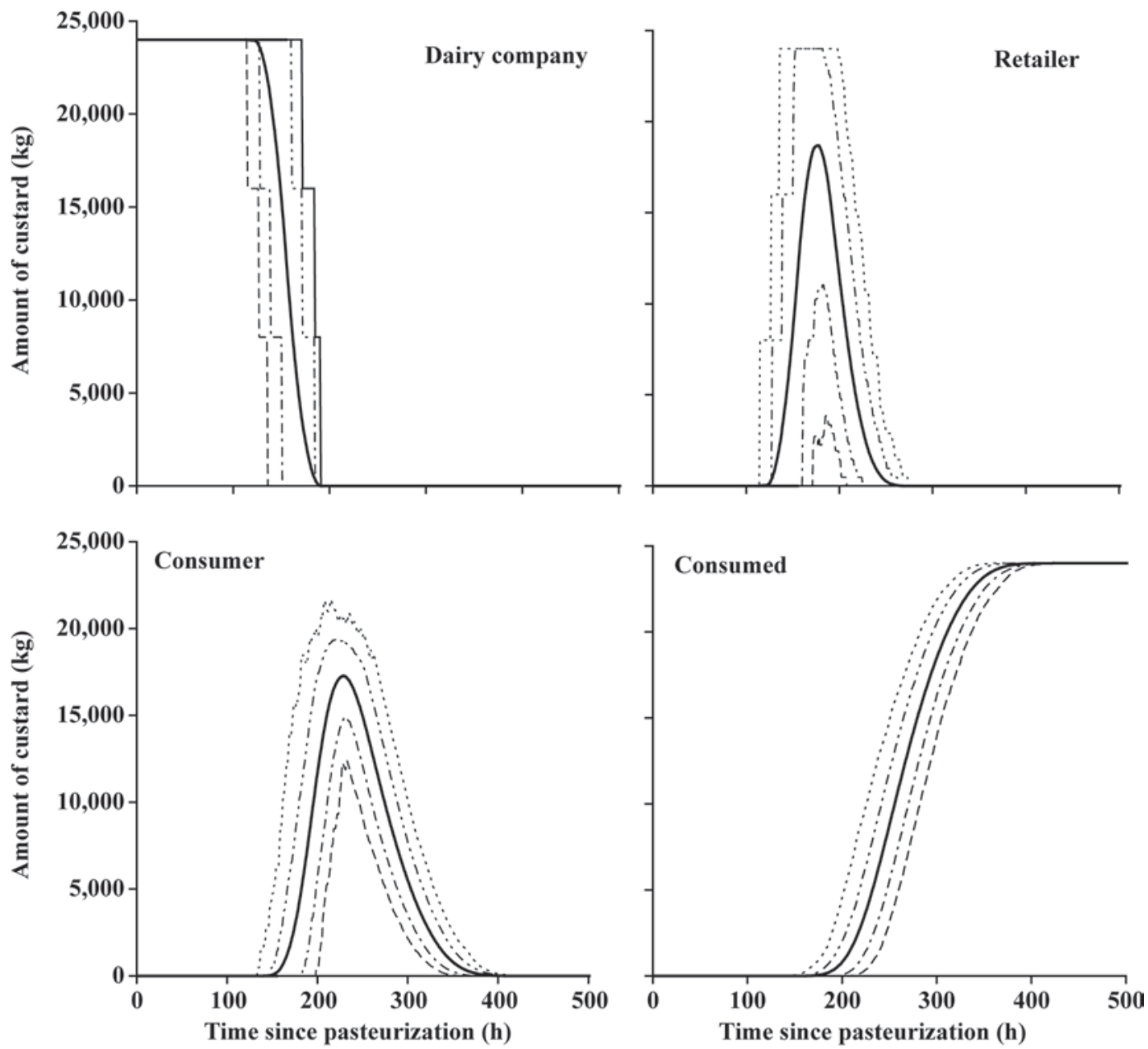

--- Minimum $\quad-\cdot-5$ th percentile $\quad$ Mean $\quad-\cdots-95$ th percentile $\cdots \cdot$ Maximum

Figure 3. Location of the amount of custard of 1 batch $(24,000 \mathrm{~L})$ over the supply chain in a situation with quarantine. Top left panel: dairy company stage, including the production site, transport, and distribution center of the dairy company; top right panel: retailer stage, including the distribution center of the retailer, transport, and stores; bottom left panel: the consumer stage; bottom right panel: the consumed amount of custard.

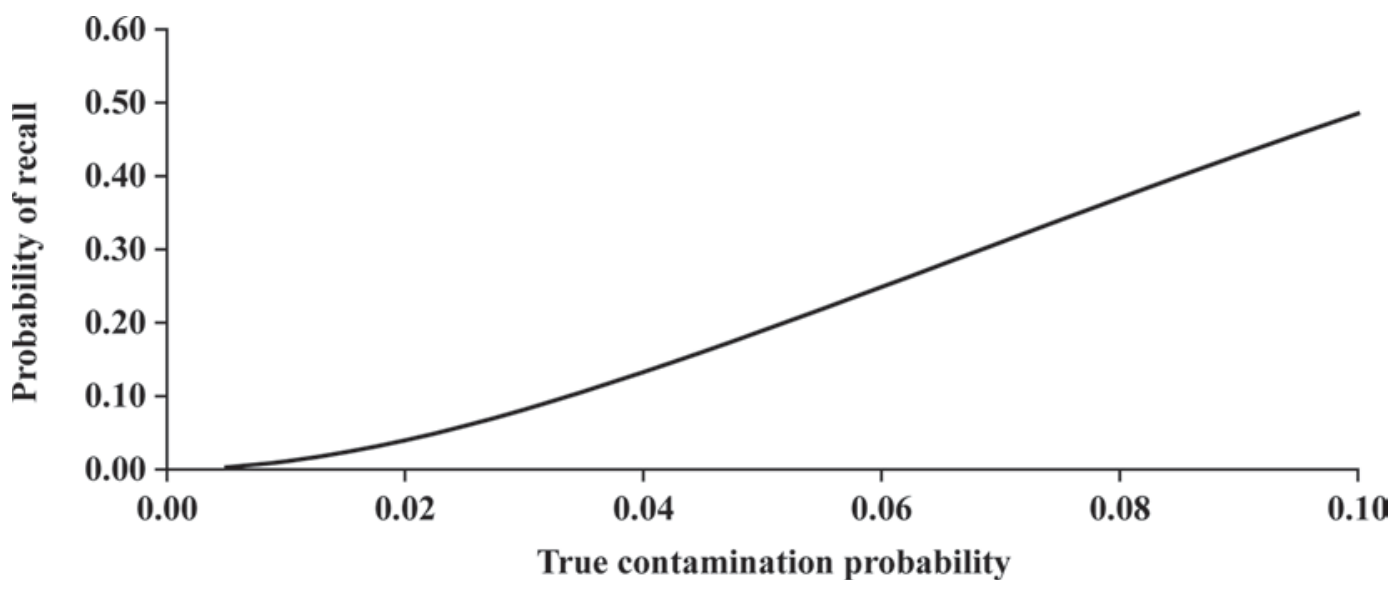

Figure 4. The probability of a recall related to the true contamination probability with Pseudomonas spp. 


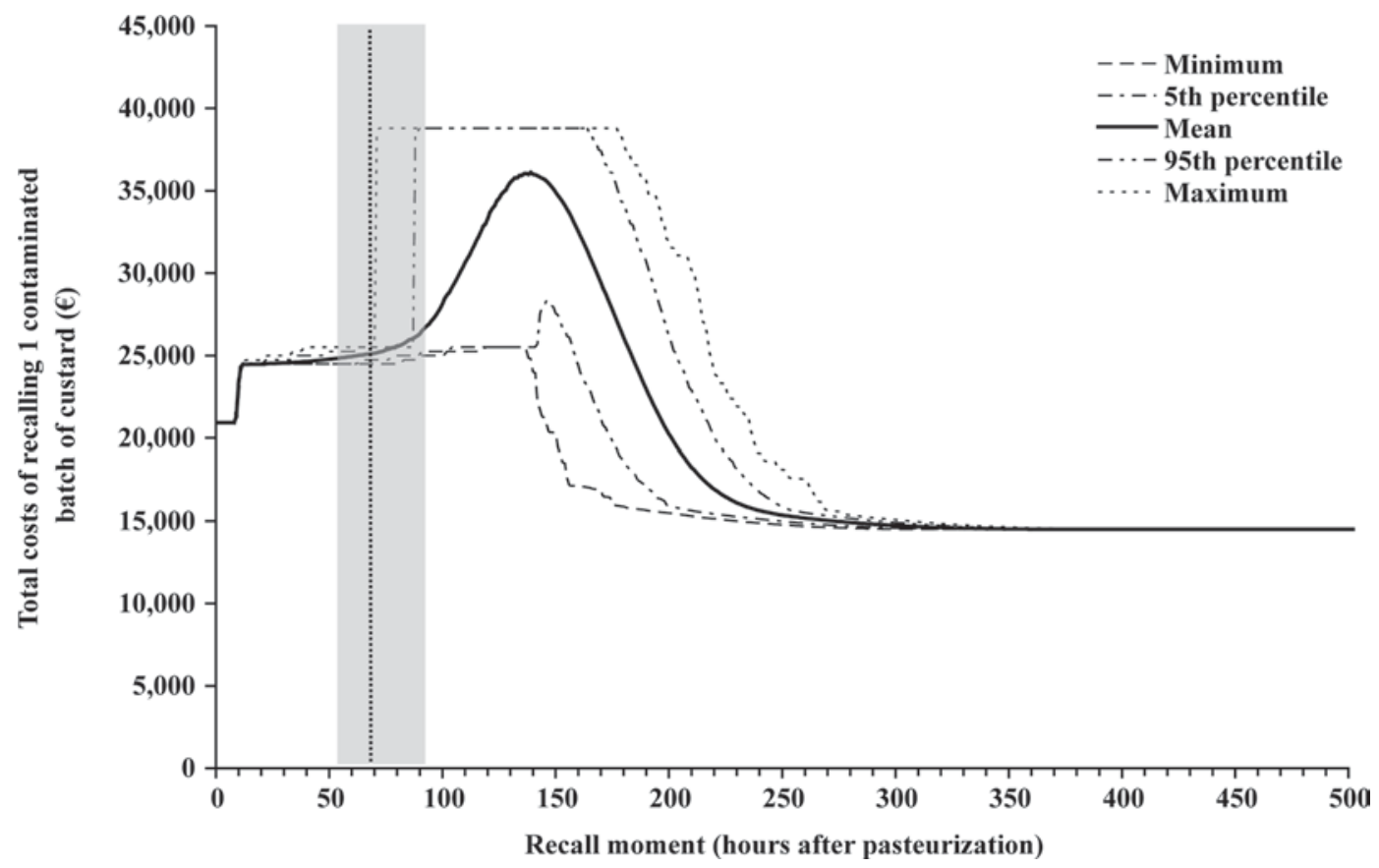

Figure 5. Net recall costs of a batch of custard contaminated with Pseudomonas spp. in relation to the recall moment (i.e., hours after pasteurization) in a situation without quarantine. The gray area indicates the period between the 5th and 95th percentiles of the moment at which the test results are available; the dashed vertical line indicates the mean value.

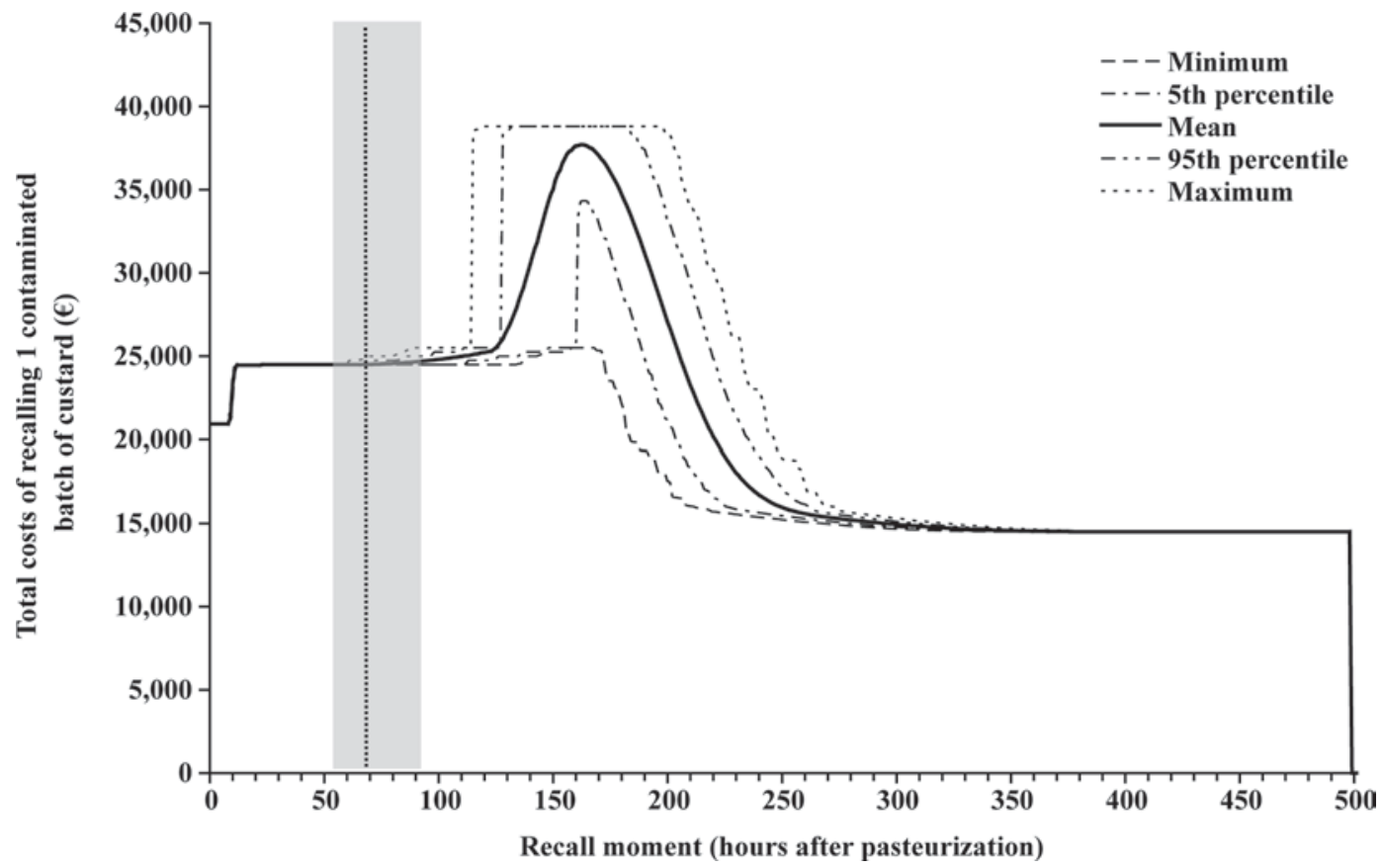

Figure 6. Net recall costs of a batch of custard contaminated with Pseudomonas spp. in relation to the recall moment (i.e., hours after pasteurization) in a situation with quarantine. The gray area indicates the period between the 5th and 95th percentiles of the moment at which the test results are available; the dashed vertical line indicates the mean value. 
the 95th percentile of time of detection. Recall moments of 125 and 155 were chosen to reflect the time frame in which contamination at the production plant with Pseudomonas spp. is likely to be detected based on consumer complaints. Consumer complaints after approximately $155 \mathrm{~h}$ are often attributable to causes of spoilage other than Pseudomonas spp. The mean $A R C$ is lower than $€ 100$ if $\operatorname{Pr}($ cont $)$ is 0.005 or $\operatorname{Pr}(P S \geq 2)$ is
0.003, whereas the $A R C$ exceeds $€ 12,000$ when $\operatorname{Pr}($ cont $)$ is 0.100 or $\operatorname{Pr}(P S \geq 2)$ is 0.485 . The recall moment has a large effect on $A R C$. A recall moment when part of a batch has just left the dairy company results in the highest costs. A recall moment when the whole batch is still at the dairy company or a substantial part of the batch is already at consumers results in the lowest costs. Quarantine has hardly an effect on the mean
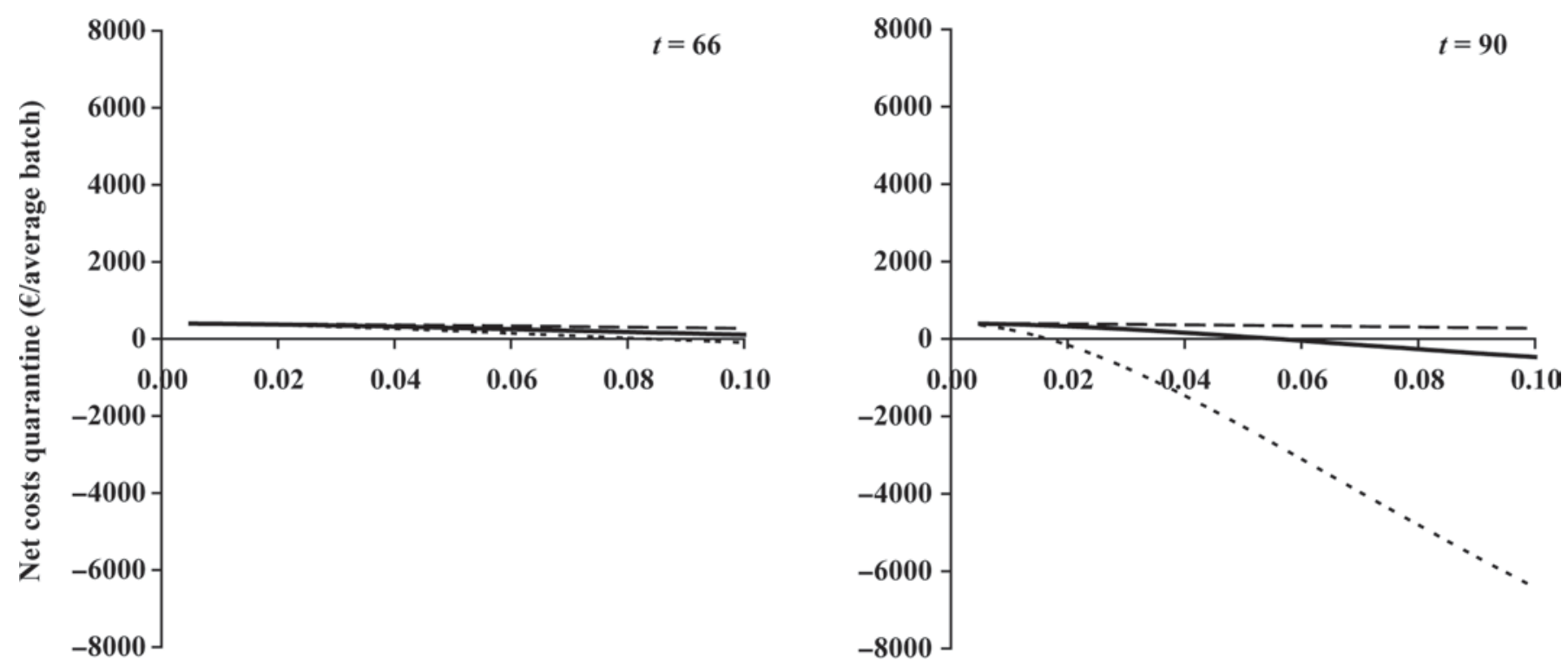

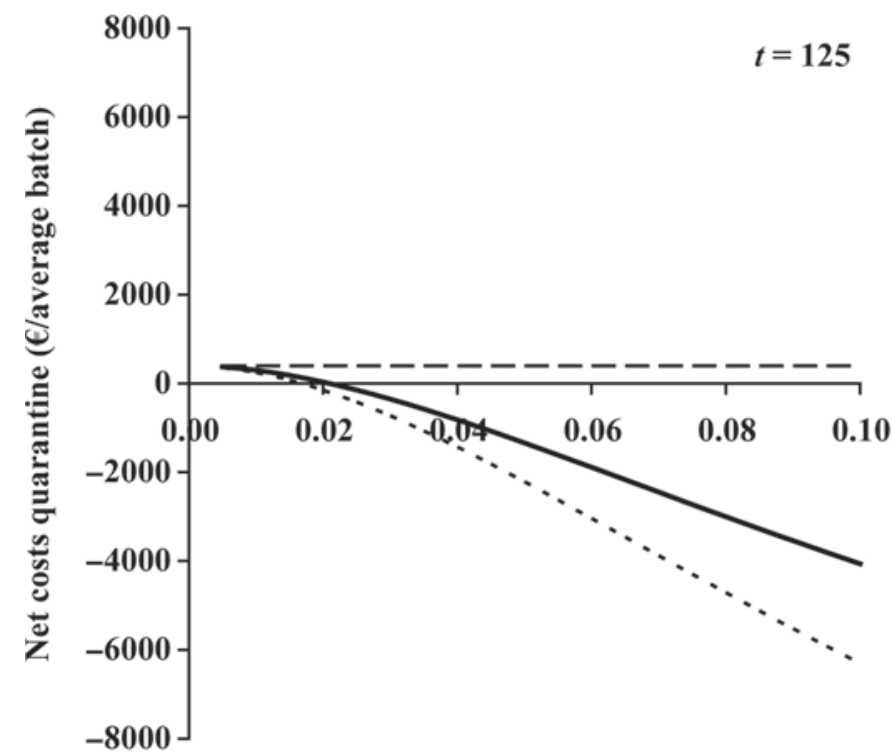

True contamination probability

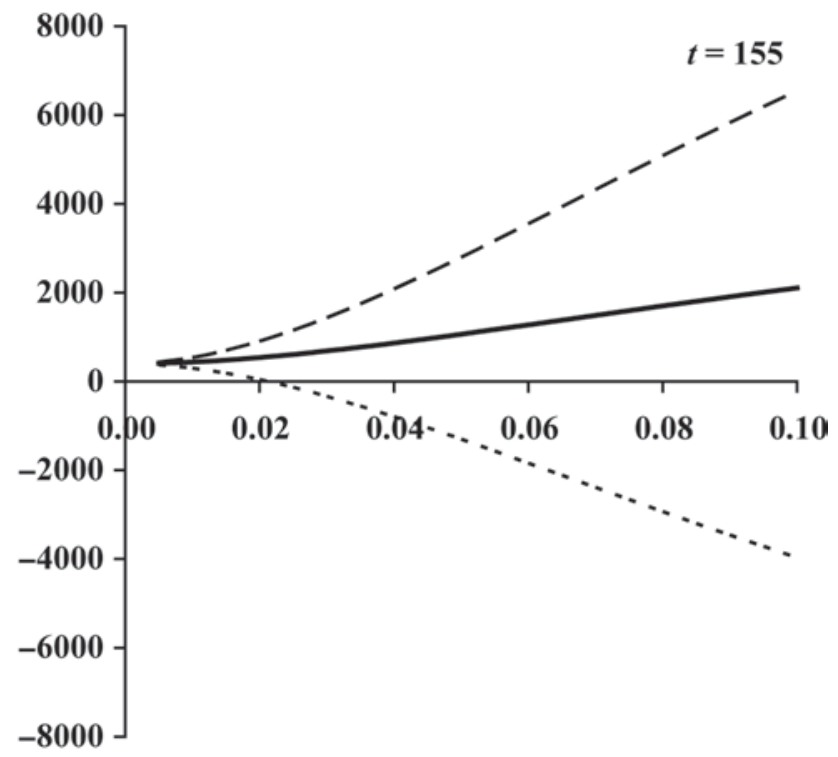

True contamination probability

\section{- Mean $\quad \cdots \cdot 5$ th percentile $\quad---95$ th percentile}

Figure 7. Mean, 5th, and 95th percentiles of the simulated net recall costs of quarantine for different recall moments depending on the true contamination probability. 
Table 4. Recall costs $(€)$ per average batch of custard produced as function of the true contamination probability $[\operatorname{Pr}($ cont $)]$ and the probability of a recall $[\operatorname{Pr}(P S \geq 2)]^{1}$

\begin{tabular}{|c|c|c|c|c|c|c|c|c|c|}
\hline \multirow[b]{3}{*}{$\operatorname{Pr}($ cont $)$} & \multirow[b]{3}{*}{$\operatorname{Pr}(P S \geq 2)$} & \multicolumn{8}{|c|}{ Time from moment first custard leaves high pasteurization (h) } \\
\hline & & \multicolumn{2}{|c|}{66} & \multicolumn{2}{|c|}{90} & \multicolumn{2}{|c|}{125} & \multicolumn{2}{|c|}{155} \\
\hline & & Mean & $95-5 \mathrm{P}$ & Mean & $95-5 \mathrm{P}$ & Mean & $95-5 \mathrm{P}$ & Mean & $95-5 \mathrm{P}$ \\
\hline & & \multicolumn{8}{|c|}{ Without quarantine } \\
\hline 0.005 & 0.003 & 72 & 2 & 75 & 38 & 100 & 38 & 96 & 36 \\
\hline 0.010 & 0.011 & 274 & 8 & 287 & 145 & 381 & 145 & 368 & 138 \\
\hline 0.015 & 0.023 & 590 & 18 & 616 & 312 & 818 & 312 & 790 & 296 \\
\hline 0.020 & 0.040 & 1,001 & 30 & 1,046 & 530 & 1,388 & 530 & 1,341 & 502 \\
\hline 0.025 & 0.059 & 1,493 & 45 & 1,561 & 791 & 2,072 & 791 & 2,001 & 749 \\
\hline 0.050 & 0.189 & 4,751 & 143 & 4,966 & 2,517 & 6,592 & 2,517 & 6,367 & 2,385 \\
\hline 0.075 & 0.340 & 8,538 & 257 & 8.925 & 4.523 & 11,846 & 4,523 & 11.442 & 4,286 \\
\hline \multirow[t]{2}{*}{0.100} & 0.485 & 12,183 & 367 & 12,736 & 6,454 & 16,903 & 6,454 & 16,327 & 6,115 \\
\hline & & \multicolumn{8}{|c|}{ With quarantine } \\
\hline 0.005 & 0.003 & 73 & 0 & 73 & 0 & 74 & 0 & 106 & 38 \\
\hline 0.010 & 0.011 & 279 & 0 & 279 & 0 & 282 & 0 & 403 & 145 \\
\hline 0.015 & 0.023 & 599 & 0 & 599 & 0 & 606 & 0 & 866 & 312 \\
\hline 0.020 & 0.040 & 1,016 & 0 & 1,016 & 0 & 1,028 & 0 & 1,470 & 530 \\
\hline 0.025 & 0.059 & 1,517 & 0 & 1,517 & 0 & 1,534 & 0 & 2,193 & 791 \\
\hline 0.050 & 0.189 & 4,826 & 0 & 4,826 & 0 & 4,881 & 0 & 6,978 & 2,517 \\
\hline 0.075 & 0.340 & 8,672 & 0 & 8.672 & 0 & 8.772 & 0 & 12.540 & 4,523 \\
\hline 0.100 & 0.485 & 12,374 & 0 & 12,374 & 0 & 12,516 & 0 & 17,893 & 6,454 \\
\hline
\end{tabular}

${ }^{1} 95-5 \mathrm{P}=$ the range between the 5 th and the 95 th percentiles.

$A R C$. However, quarantine reduces the range between the 5 th and 95th percentiles. For recall moments up to and including $t=125$, it reduces the range to zero.

\section{Net Recall Costs of Applying Quarantine}

Figure 7 summarizes the net recall costs if a quarantine situation is in place $\left(\boldsymbol{N} \boldsymbol{C}_{q}\right)$. The mean $N C_{q}$ is positive (costs exceed benefits) for $\operatorname{Pr}($ cont) values up to and including 0.020 for the recall moments indicated. In contrast, mean $N C_{q}$ is negative (quarantine can be profitable) for relatively high $\operatorname{Pr}($ cont $)$ at $t=90$ and $t=125$. However, in all these cases the $95 \% N C_{q}$ is positive, indicating that it is possible that quarantine is not profitable.

\section{DISCUSSION}

The relation between the moment at which a recall of a batch of Dutch custard is initiated because of spoilage with Pseudomonas spp. and the direct costs of the recall was analyzed. Furthermore, the consequences of a quarantine of $48 \mathrm{~h}$ after testing a batch on the net recall costs were quantified. A simulation approach was used because the distribution of a batch of custard over the chain over time is not fixed. We showed that the net recall costs depend on the moment a recall is initiated. This moment determines the distribution of a batch of custard along the supply chain. The net recall costs increase with the recall moment but decrease after reach- ing a maximum of about $€ 36,500$ because more and more custard is consumed. However, the indirect recall cost may increase with the amount of custard that is consumed. A later recall moment increases the risk of damaging consumer confidence in the specific type of custard, the company's name, or the whole sector, as has been observed in consumer milk by Velthuis et al. (2009). Further research is needed to include the indirect recall costs in a model to quantify the relationship between the recall moment and corresponding cost.

Another unknown effect is the effect of multiple recalls or multiple purchases of a defective product on consumer confidence. Only a limited number of consumers have asked for a refund (i.e., 0.13\%), but what is the real effect on their purchase strategy? Whereas a single recall might not have a big negative effect, multiple recalls or multiple purchases of a defective product could have a significant effect on consumer behavior. More research is needed on this point.

Quantification of how recall costs vary with the moment a recall is initiated provides insight as to where innovative testing, monitoring, traceability, and management systems can be located and what their possible effects are. When considering new systems along the custard chain and the probability of having a recall, the costs and benefits of a new system should be taken into account as well.

The $\operatorname{Pr}($ cont $)$ can be difficult to calculate in practice because it includes both the incidental postprocessing contamination incidents and possible biofilm incidents. 
The latter can lead to contamination probabilities of 0.2 to 0.5 , increasing the $\operatorname{Pr}($ cont $)$ to high values (Langeveld et al., 1995; Brooks and Flint, 2008).

Although this research focuses on recalls based on spoilage problems that are detected with the end product testing, it includes situations in which, despite the end product testing, consumers experience spoilage and contact the consumer care line. These incidents can occur from approximately $100 \mathrm{~h}$ after pasteurization up to the end of shelf life in the situation without quarantine and from $130 \mathrm{~h}$ after pasteurization in a situation with quarantine.

The results of this study can also be used in the decision whether to invest in a faster test protocol for end product testing. For example, if the time of detection can be reduced by $24 \mathrm{~h}$ the 95 th percentile of the recall costs in a nonquarantine situation is reduced significantly. However, the mean of the recall costs is not reduced by the improvement. In a 48-h quarantine situation, a faster test protocol does not reduce the mean or 95th percentile of the total recall costs. Increasing the speed of the test by using a new test and thereby having the results faster can reduce quarantine period and associated costs, which were assumed to be fixed in the current study.

The testing protocol currently in use takes between 2 and 4 d. Faster tests (e.g., the fluorescence in situ hybridization method) might be used to detect and count Pseudomonas spp. in milk in $2 \mathrm{~h}$. The sensitivity of this method is good but could be increased by combining it with membrane filtration (Gunasekera et al., 2003). However, this test is more expensive. Additional research is needed to determine the economic optimal quality control protocol because the costs of less optimal logistical process or another test should outweigh the benefits of having the information more quickly.

It is important for the individual food producers to study the different options in quality management in relation to the economic impact of recalling batches that are suspected based on end product testing. With this study we show that quality assurance decisions can be supported by decision models that include not only technical but also economic information.

\section{ACKNOWLEDGMENTS}

This study was partially funded by the KB6 theme Food Safety of Wageningen University and Research Center (Wageningen, the Netherlands). We gratefully acknowledge Suzan Horst and Rien Habraken (Koninklijke FrieslandCampina NV) with respect to the information provided.

\section{REFERENCES}

Brooks, J. D., and S. H. Flint. 2008. Biofilms in the food industry: Problems and potential solutions. Int. J. Food Sci. Technol. 43:2163-2176.

Dijkhuizen, A. A., and R. S. Morris. 1997. Animal Health Economics. 1st ed. Postgraduate Foundation in Veterinary Science, University of Sydney, Sydney, Australia.

Dorp, C. A. v. 2004. Reference-data modelling for tracking and tracing. Page 240 in Information Technology Group. PhD thesis, Wageningen University, Wageningen, the Netherlands.

Dupuy, C., V. Botta-Genoulaz, and A. Guinet. 2005. Batch dispersion model to optimise traceability in food industry. J. Food Eng. 70:333-339.

EC. 2002. Regulation (EC) No. 178/2002 of the European Parliament and of the Council. Page 24. Vol. 178/2002. Official Journal of the European Communities, European Commission, Brussels, Belgium.

Eneroth, A., S. Ahrne, and G. Molin. 2000. Contamination of milk with Gram-negative spoilage bacteria during filling of retail containers. Int. J. Food Microbiol. 57:99-106.

Eneroth, A., A. Christiansson, J. Brendehaug, and G. Molin. 1998. Critical contamination sites in the production line of pasteurised milk, with reference to the psychrotrophic spoilage flora. Int. Dairy J. 8:829-834.

Gunasekera, T. S., M. R. Dorsch, M. B. Slade, and D. A. Veal. 2003. Specific detection of Pseudomonas spp. in milk by fluorescence in situ hybridization using ribosomal RNA directed probes. J. Appl. Microbiol. 94:936-945.

Huck, J. R., M. Sonnen, and K. J. Boor. 2008. Tracking heat-resistant, cold-thriving fluid milk spoilage bacteria from farm to packaged product. J. Dairy Sci. 91:1218-1228.

Kramer, M. N., D. Coto, and J. D. Weidner. 2005. The science of recalls. Meat Sci. 71:158-163.

Langeveld, L. P. M., R. M. G. E. van Montfort-Quasig, A. H. Weerkamp, R. Waalewijn, and J. S. Wever. 1995. Adherence, growth and release of bacteria in a tube heat exchanger for milk. Neth. Milk Dairy J. 49:207-220.

Meuwissen, M. P. M., A. G. J. Velthuis, H. Hogeveen, and R.B.M. Huirne., 2003. Traceability and certification in meat supply chains. J. Agribusiness 21:167-181.

Meuwissen, M. P. M., A. L. A. Van Andel, M. A. P. M. Van Asseldonk, and R. B. M. Huirne. 2009. Eliciting processing industry damage from feed crises. Br. Food J. 111:878-892.

Palisade-Corporation. 2004. @RISK. 4.5 ed. Palisade Corporation, Newfield, NY.

Salin, V., N. H. Hooker, and R. Teratanavat. 2005. Recall event timing: Measures of managerial performance in U.S. meat and poultry plants. Agribusiness 21:351-373.

SkeesJ. R.BottsA.ZeuliK.A. 2001. The potential for recall insurance to improve food safety. Int. Food Agribusiness Manage. Rev. 4:99 111

Teratanavat, R., and N. H. Hooker. 2004. Understanding the characteristics of US meat and poultry recalls: 1994-2002. Food Contr. 15:359-367.

VelthuisA. G. J.MeuwissenM.HuirneR. B. M. 2009. Distribution of direct recall costs along the milk chain. Agribusiness 25:466-479.

Vose, D. J. 2000. Risk Analysis: A Quantitative Guide. 2nd ed. John Wiley \& Sons, Hoboken, NJ. 